\title{
Temperature derivative of the resistance of spin valves
}

\author{
Simon Granville ${ }^{\mathrm{a}, *}$, Haiming Yu ${ }^{\mathrm{a}, \mathrm{b}}$, Julie Dubois ${ }^{\mathrm{a}, \mathrm{c}}$, Laurent Gravier ${ }^{\mathrm{a}}$, Jean-Philippe Ansermet ${ }^{\mathrm{a}}$ \\ a Institut de Physique des Nanostrutures, Ecole Polytechnique Fédérale de Lausanne, Station 3, CH-1015 Lausanne, Switzerland \\ b State Key Laboratory for Mesoscopic Physics, School of Physics, Peking University, Beijing 100871, People's Republic of China \\ ${ }^{\text {c } E c o l e ~ d e s ~ M i n e s, ~} 60$ bd Saint Michel 75006 Paris, France
}

\section{A R T I C L E I N F O}

Available online 21 March 2009

Keywords:

Spin torque

Nanostructure

Spin valves

Magnetoresistance

\begin{abstract}
A B S T R A C T
Spin-dependent transport properties of nanowires containing individual $\mathrm{Co} / \mathrm{Cu} / \mathrm{Co}$ pseudo-spin valves were investigated by monitoring an $\mathrm{AC}$ voltage induced by a temperature oscillation under a continuous DC current. We observed sharp peaks in the field-dependent AC voltage response. These peaks only appear close to the magnetic field range where conventional magnetoresistance shows a reversible and gradual transition. The effect is interpreted as an extra dissipation process with a distinct temperature dependence that occurs in non-collinear magnetic configurations when transverse moment relaxation takes place.
\end{abstract}

\section{Introduction}

Spin-dependent transport in magnetic multilayers plays an important role in spintronics and has already demonstrated its significance in effects such as giant magnetoresistance (GMR) $[1,2]$. In our research, spin-dependent transport is probed by a novel measurement protocol called magneto-thermogalvanic voltage (MTGV), where we monitor the AC voltage response induced by an oscillating heat source under a constant DC current, revealing for example, spin-dependent Peltier [3] and Seebeck effects [4]. We also have applied this measurement to thin films containing magnetic clusters [5] and to nanowires containing five equally spaced $\mathrm{Co} / \mathrm{Cu}$ bilayers [6]. In this paper, we apply this measurement technique to $\mathrm{Co} / \mathrm{Cu} / \mathrm{Co}$ pseudo-spin valves (PSV) with the current perpendicular to the plane of the layers (CPP). By measuring the magnetic field dependence of the signal, we observed a pronounced response which we attribute to spintorque effects due to non-collinear magnetic configurations.

\section{Experimental}

We synthesize $\mathrm{Cu}$ nanowires containing $\mathrm{Co} / \mathrm{Cu} / \mathrm{Co}$ PSV structures in nanoporous polymer templates by electrodeposition. The pores are about $6 \mu \mathrm{m}$ long with a diameter of $40-60 \mathrm{~nm}$. Gold layers are sputtered on both sides of the membrane, with the bottom gold layer used as the working electrode during the electroplating. The top layer is thin enough that the pores are not

\footnotetext{
* Corresponding author. Tel.: +41216934412; fax: +41216933604.

E-mail address: simon.granville@epfl.ch (S. Granville).
}

completely blocked, so that we can grow nanowires in the pores [7]. Most importantly, by monitoring the deposition current, we observe a sharp jump when a nanowire grows to the top of the membrane and contacts the thin gold layer. With this technique, we can achieve single nanowire contact simply by stopping deposition as soon as we observe the sudden current jump. We electroplate in a single bath containing both $\mathrm{Cu}$ and $\mathrm{Co}$. In the previous work we have found that the Co layers contain about 15\% $\mathrm{Cu}$, and have the fcc structure [8]. Our PSV structures typically consist of 10 and $30 \mathrm{~nm}$ layers of Co, with a $10 \mathrm{~nm}$ Cu layer in between, as shown in Fig. 1.

Confirmation that the samples with PSV structures are successfully prepared is provided by GMR: a singly contacted $\mathrm{Cu}$ nanowire with one $\mathrm{Co} / \mathrm{Cu} / \mathrm{Co}$ PSV demonstrates two jumps in the resistance as the field makes a single complete sweep. These jumps correspond to switching between parallel and anti-parallel magnetic configurations of the two Co layers. Switching fields in these layers are known to be broadly distributed [9]. In order to increase the production yield of samples with desired switching events, we sometimes grow nanowires containing several PSVs in series, sufficiently separated from each other (more than $1 \mu \mathrm{m}$ apart) so that the magnetic couplings between PSVs remain insignificant.

The magnetoresistance (MR) of the nanowires was measured by conventional detection of the AC voltage due to a 1-100 $\mu \mathrm{A} A C$ current (at $<1 \mathrm{kHz}$ ) applied in the CPP geometry. The measurement protocol for MTGV is explained as follows: a chopped laser beam irradiates one end of the nanowire, which forces the temperature to oscillate at the chopping frequency. The corresponding AC voltage across the sample is measured by lock-in detection under a steady DC current $I_{\mathrm{DC}}$. Hence, the MTGV measurement is equivalent to a lock-in detection of $\mathrm{d} R / \mathrm{d} T$ as a 


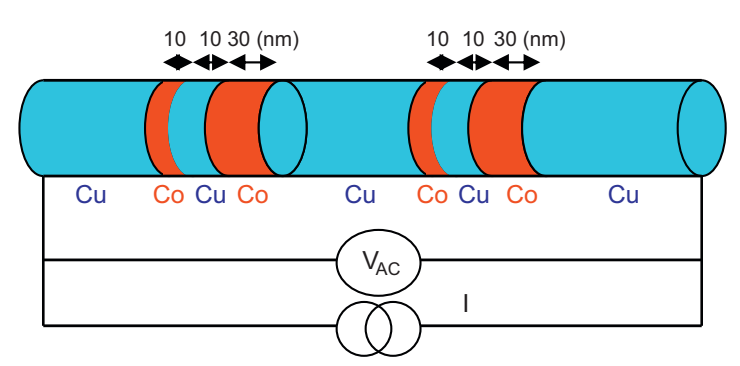

Fig. 1. (Color online) Schematic picture of the investigated structures: a few PSV structures $(\mathrm{Co} / \mathrm{Cu} / \mathrm{Co})$ are embedded in a $\mathrm{Cu}$ nanowire. A laser beam causes a temperature oscillation of the nanowire. VAC is measured by lock-in detection.

function of the applied field, $H$. We measure the field dependence of the signal with magnetic field applied perpendicular or parallel to the wire axis. The maximum magnetic field applied was $\pm 0.8 \mathrm{~T}$. All MTGV measurements reported here were carried out at room temperature with a laser chopping frequency of $22 \mathrm{~Hz}$ and an applied $I_{\mathrm{DC}}$ of $100 \mu \mathrm{A}$. The thermoelectric contribution is negligible compared to the MTGV signals observed at this current [6]. The baseline of the measurements is due to the temperature dependence of the resistivity of the $\mathrm{Cu}$ nanowires.

\section{Results and discussion}

We performed the MR and MTGV measurements in a $\mathrm{Cu}$ nanowire containing five $\mathrm{Co} / \mathrm{Cu} / \mathrm{Co}$ PSVs $(10 \mathrm{~nm} / 10 \mathrm{~nm} / 30 \mathrm{~nm})$ in series, separated by several microns within the nanowire (Fig. 2). The external magnetic field is applied parallel to the wire axis. The steps in MR appear between magnetic fields of $\pm 300 \mathrm{mT}$ indicating the switching fields of the individual PSVs. The switches include sharp jumps as well as more gradual gradients of the resistance, indicating some PSVs are more pinned in the field-parallel-to-wire geometry than others. In the MTGV measurement, several sharp peaks are observed in the same magnetic field range as the MR changes. Outside of this field range the MTGV is flat and unremarkable. The largest peak is about $4.3 \%$, appearing over a constant baseline of $6.9 \mu \mathrm{V}$, which provides an MTGV ratio greater than that of the GMR ratio of any PSV. Some peaks occur at fields where GMR is flat, hence the peaks are not simply due to large values of $\mathrm{d} R / \mathrm{d} H$.

However, by comparison of the GMR to the MTGV in Fig. 2, it is clear that MTGV peaks arise from processes occurring near fields where the PSV layers switch in a smooth fashion. This correlation between the MR behaviour and the MTGV peaks can be more easily identified in Fig. 3, which presents both measurements carried out on a sample containing only two isolated PSVs inside a nanowire. The MR clearly shows four switches in a single magnetic field sweep: two reversible transitions over a range of field values and two irreversible transitions. The apparent steps in GMR at $\pm 100 \mathrm{mT}$ are artifacts in the measurement. In the MTGV measurement only two distinctive peaks over the constant baseline of $1.26 \mu \mathrm{V}$ are seen, of about $4 \%$ and $6 \%$, respectively, which shows the higher sensitivity of the MTGV technique compared with the total MR ratio of about $0.2 \%$. The peaks in MTGV exclusively appear near the reversible magnetization reversal mode at magnetic fields around $\pm 72 \mathrm{mT}$. These reversals in MR are those occurring over a larger field range. To the contrary, near the sharp MR changes around $\pm 35 \mathrm{mT}$, there are no MTGV peaks visible out of the noise level.

The comparison of the MTGV measurement to the MR suggests that the MTGV peaks are provoked by non-collinear magnetic configurations of the two Co layers in a PSV. These peaks are very sensitive to the magnetic field, and the typical full-width at half-

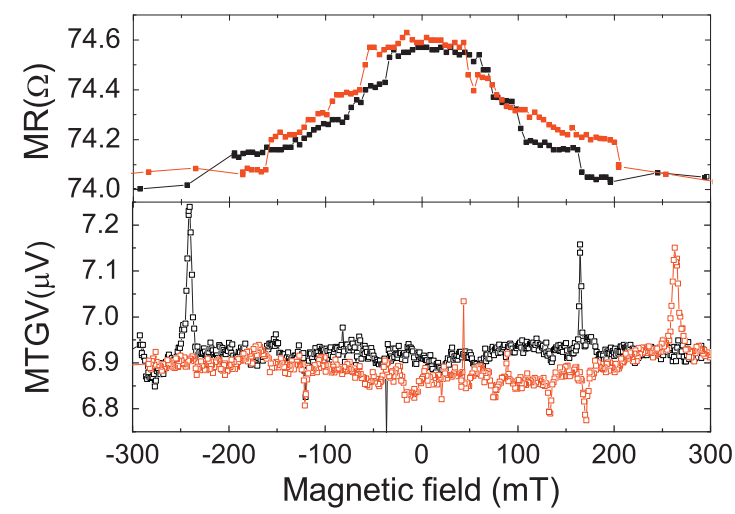

Fig. 2. (Color online) MR and MTGV of a Cu nanowire containing five isolated Co/ $\mathrm{Cu} / \mathrm{Co}$ pseudo-spin valves in series, with magnetic field applied parallel to the nanowire, ramping field up (black) and down (red). (For interpretation of the references to colour in this figure legend, the reader is referred to the web version of this article.)

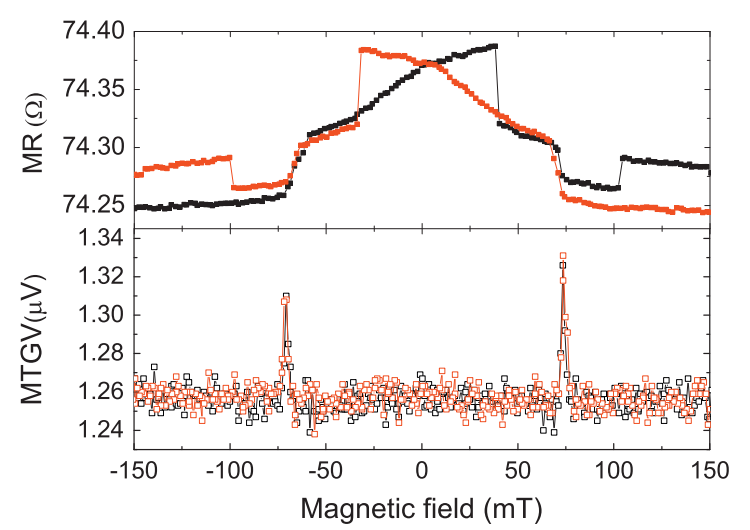

Fig. 3. (Color online) MR and MTGV of a $\mathrm{Cu}$ nanowire containing two $\mathrm{Co} / \mathrm{Cu} / \mathrm{Co}$ pseudo-spin valves in series, with magnetic field applied parallel to the nanowire, ramping field up (black) and down (red). (For interpretation of the references to colour in this figure legend, the reader is referred to the web version of this article.)

maximum is only about $3 \mathrm{mT}$. This indicates the MTGV peaks are only sparked in a particular magnetic configuration during the switching process. By comparison with the GMR curves measured in multiple samples, there is no significant peak observed in MTGV when there is an abrupt jump in MR, at the point of a sudden switch of a PSV.

The pronounced peaks are only detected when the transition is smooth and reversible, which means the switching goes through a configuration where a high dissipation process occurs.

The sharp MTGV peaks observed in this instance confirm previous studies on the MTGV of five $\mathrm{Co} / \mathrm{Cu}$ bilayers [6]. They are not due to the previously observed spin-dependent Peltier effect [3] since the temperature change over the thickness of one bilayer is extremely small. It has been shown that experimental data on CPPMR as a function of the angle $\theta$ between the magnetization orientations in the layers of spin valves can be cast in the form [10]

$\frac{R(\theta)-R(0)}{R(\pi)-R(0)}=\frac{1-\cos ^{2}(\theta / 2)}{1+\chi \cos ^{2}(\theta / 2)}$

The observed MTGV voltage can be deduced as $V(\theta)=I_{D C} T_{A C}$ $d R(\theta) / d T$, where $T_{A C}$ is the amplitude of the temperature oscillation. $V(0)$ yields $T_{A C}$ for the nanowire. We now show that the MTGV interpreted in terms of (1) must arise from the temperature dependence of $\chi$ and that a temperature-induced change in magnetocrystalline anisotropy will not significantly affect the 


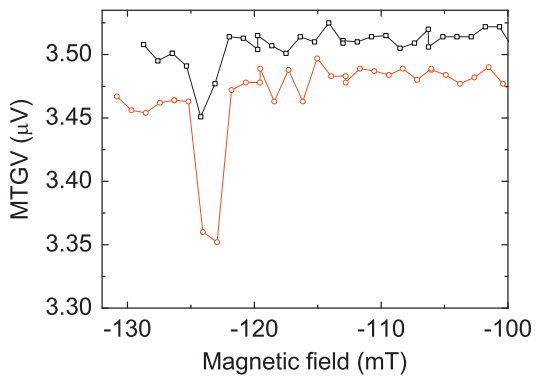

Fig. 4. (Color online) MTGV of a $\mathrm{Cu}$ nanowire containing one $\mathrm{Co} / \mathrm{Cu} / \mathrm{Co} \mathrm{PSV}$, with magnetic field applied perpendicular to the nanowire, without (black) and with (red) a transverse field of $2 \mathrm{mT}$. The transverse field enhances the MTGV peak by a factor of 2. (For interpretation of the references to colour in this figure legend, the reader is referred to the web version of this article.)

voltage measured by the way of $d R(\theta) / d T$. In a worst case estimate of this spurious effect, we consider the magnetization angle of only one layer to be affected by heating. The switching field of a layer is the vector sum of the anisotropy $H_{a}$ and dipolar $H_{\text {dip }}$ fields, and we consider these fields to be transverse to each other, which corresponds to the configuration most sensitive to a reduction in $H_{a}$. The laser heating of $\sim 1 \mathrm{~K}$ will reduce $H_{a}$ of the fcc Co by $0.6 \%$ at room temperature [11], while $H_{\text {dip }}$ will remain constant. This results in a change in angle $\Delta \theta$ where $(1 / \Delta \theta) d \theta / d T=H_{d i p} / H_{a}$, assuming $\Delta H_{a} / H_{a}=\Delta \theta / \theta$, for a MTGV voltage $V=1 \times 10^{-4}$ $\mathrm{A} \cdot 1 \mathrm{~K} \cdot(\Delta \theta \partial R / \partial \theta) \cdot H_{\text {dip }} / H_{a} \cdot(\Delta \theta \partial R / \partial \theta)<0.01 \cdot \Delta R$ for $\chi<50$ (see discussion of this parameter below), and using $\Delta R=0.05 \Omega$ and $H_{\text {dip }} /$ $H_{a}=0.1$ for our PSVs, we find $V=5 \mathrm{nV}$, an order of magnitude smaller than the detected MTGV voltage peaks. Therefore, the measured response is due to a different mechanism than the temperature dependence of the magnetocrystalline anisotropy.

The sharp peaks that occur only when the MR switch covers a range of $\sim 7 \mathrm{mT}$ suggest instead a transport effect associated with the spin-transfer torque, which is effective only in non-collinear orientations of the Co layers of each PSV. Further evidence of the importance of the magnetic configuration on the MTGV response can be seen, for example, in the observation of Fig. 4. Here, the nanowire contains a single PSV, the main applied field is in the plane of the layers, and an additional small field is applied perpendicular to both the nanowire and to the main ramping field. The addition of such a small transverse field was found to enhance the MTGV response, by a factor of two or more in all samples tested. It is likely that the transverse field eases access to non-collinear configurations.

By calculating the temperature derivatives in (1) and rearranging the terms, we find

$\frac{1}{\chi} \frac{d \chi}{d T}=\frac{\operatorname{MTGV}}{\operatorname{GMR}}\left(\frac{1}{\rho} \frac{d \rho}{d T}\right)\left(\chi \frac{d R_{n}\left(\theta_{\max }\right)}{d \chi}\right)^{-1}$

We use the symbol MTGV for $V\left(\theta_{\max }\right) / V(0)-1$, and GMR for $R(\pi) /$ $R(0)-1 . \rho$ is the resistivity of the PSV and $\theta_{\max }$ the angle of the maximum $d R_{n}(\theta) / d \chi$. The last coefficient of (2) turns about to be $\approx 4$ for $\chi>3$.

The interpretation of $\chi$ is model dependent. For Shpiro et al. [12], it is directly linked to the length over which the transverse moment of the conduction electron-spins decay, and this length depends on the square root of the conductivity. Brataas et al. express $\chi$ in terms of the ratio of the interface mixing conductance to the conductivity [13]. As shown in Fig. 4, a small transverse field can increase the measured MTGV by a factor of two, indicating the relative magnetic orientations of the two Co layers in our PSV samples are very sensitive to small perturbations. MTGV data from spin valves where a high level of control over the magnetic configurations can be exerted, e.g. in exchange-biased spin valves, are needed before experimental results can be compared directly with theory. Another requirement is the very accurate resistivity values in order to separate the contribution from the $\mathrm{Cu}$ nanowire. Additionally, further developments in the models may allow more straightforward interpretations of $\chi$ from this type of experiment [14].

\section{Conclusion}

We have performed MR and MTGV measurements on nanowires with $\mathrm{Co} / \mathrm{Cu} / \mathrm{Co}$ pseudo-spin valve structures. We showed that sharp peaks are observed in the MTGV profile only when the magnetization switching is reversible and occurs over a finite field range. This effect reveals a dissipative process with a temperature dependence much larger than that of the resistivity of the homogeneous metal. The peaks occur when the Co layer magnetizations are forced to turn out of alignment with one another. This suggests that the dissipative mechanism is the decay of the transverse moment associated with the spin-transfer torque.

\section{Acknowledgment}

H.Y. gratefully acknowledges the exchange agreement between Peking University and EPFL.

\section{References}

[1] M.N. Baibich, J.M. Broto, A. Fert, F. Nguyen Van Dau, F. Petroff, P. Eitenne G. Creuzet, A. Friederich, J. Chazelas, Phys. Rev. Lett. 61 (1988) 2472.

[2] G. Binasch, P. Grünberg, F. Saurenbach, W. Zinn, Phys. Rev. B 39 (1989) 4828

[3] L. Gravier, S. Serrano-Guisan, F. Reuse, J.-Ph. Ansermet, Phys. Rev. B 73 (2006) 052410.

[4] L. Gravier, A. Fábián, A. Rudolf, A. Cachin, K. Hjort, J.-E. Wegrowe, J.-Ph Ansermet, J. Magn. Magn. Mater. 271 (2004) 153.

[5] S. Serrano-Guisan, G. di Domenicantonio, M. Abid, J.-P. Abid, M. Hillenkamp, L. Gravier, J.-Ph. Ansermet, Ch. Félix, Nat. Mater. 5 (2006) 730.

[6] S. Serrano-Guisan, L. Gravier, J.-Ph. Ansermet, Mater. Sci. Eng. B 126 (2006) 292.

[7] J.-E. Wegrowe, S.E. Gilbert, D. Kelly, B. Doudin, J.-Ph. Ansermet, IEEE Trans. Magn. 34 (1998) 903.

[8] B. Doudin, J.-E. Wegrowe, S.E. Gilbert, V. Scarani, D. Kelly, J.P. Meier, J.-Ph Ansermet, IEEE Trans. Magn. 34 (1998) 968.

[9] B. Doudin, A. Blondel, J.-Ph. Ansermet, J. Appl. Phys. 79 (1996) 6090.

[10] S. Urazhdin, R. Loloee, J.W.P. Pratt, Phys. Rev. B 71 (2005) 100401.

[11] J. Goddard, J.G. Wright, Br. J. Appl. Phys. 16 (1965) 1251

[12] A. Shpiro, P.M. Levy, S. Zhang, Phys. Rev. B 67 (2003) 104430.

[13] A. Brataas, G.E.W. Bauer, P.J. Kelly, Phys. Rep. 427 (2006) 157.

[14] J. Dubois, J.-Ph. Ansermet, Phys. Rev. B 78 (2008) 184430. 